\title{
Determinants of brachial-ankle pulse wave velocity in a Japanese population: a cohort study.
}

\section{$\operatorname{AUTHOR}(\mathrm{S})$ :}

Mitani, Satoko; Fujita, Masatoshi; Shigeta, Masako; Kuriyama, Nagato; Ozaki, Etsuko; Yoshikawa, Aya; Matsui, Daisuke; Watanabe, Isao; Inoue, Kaoru; Watanabe, Yoshiyuki

\section{CITATION:}

Mitani, Satoko ... [et al]. Determinants of brachial-ankle pulse wave velocity in a Japanese population: a cohort study.. Blood pressure 2012, 21(6): 338-344

\section{ISSUE DATE:}

2012-12

URL:

http://hdl.handle.net/2433/182073

\section{RIGHT:}

(C) 2012 Informa Healthcare; This is not the published version. Please cite only the published version.; この論文は出版社版でありません。引 用の際には出版社版をご確認ご利用ください。 
Determinants of baPWV Mitani S

Title page

$<$ Title>

Determinants of brachial-ankle pulse wave velocity in Japanese population : a cohort study

\section{<Authors>}

Satoko Mitani, RN., PhD. (Corresponding author) (1)

Masatoshi Fujita, MD., PhD (2) mfujita@kuhp.kyoto-u.ac.jp

Masako Shigeta, MD., PhD. (3) mshige@koto.kpu-m.ac.jp

Nagato Kuriyama, MD., PhD. (3) nkuriyam@koto.kpu-m.ac.jp

Etsuko Ozaki, RD., MEd (3) ozaki@koto.kpu-m.ac.jp

Aya Yoshikawa (3) aya-y@koto.kpu-m.ac.jp

Daisuke Matsui, MD., PhD.(3) d-matsui@koto.kpu-m.ac.jp

Isao Watanabe, MD.(3) ricky@koto.kpu-m.ac.jp

Kaoru Inoue (3) kaoruino@koto.kpu-m.ac.jp

Yoshiyuki Watanabe, MD., PhD. (3)watanabe@koto.kpu-m.ac.jp

(1) Unit for Liveable Cities, Kyoto University Graduate School of Medicine

(2) Human Health Sciences, Kyoto University Graduate School of Medicine

(3) Kyoto Prefectural University of Medicine

$<$ Running title $>$

Determinants of baPWV

$<$ Full address of corresponding author>

Address : 53 Shogoin-Kawaharacho, Sakyo-ku, Kyoto, 606-8507, Japan

Tel: +81-75-751-3914

Fax: +81-75-751-3914

e-mail: mitani-satoko@hs.med.kyoto-u.ac.jp

$<$ The number of words>

3448 words (from Introduction to the end including Reference)

$<$ Former oral/poster presentations $>$

1) A comparison of determinants of lifestyle on augmentation index and brachial pressure. 18 Jun. 2010. European Meeting on Hypertension, Oslo, Norway 
Determinants of baPWV Mitani S

2) Characteristics of PWV and AI among various stage of hypertension 23 May, 2010. The Pulse of Asia in 2010, Tokyo, Japan 
Determinants of baPWV Mitani S

\section{Abstract}

Arterial stiffness is one of the biggest predictors of coronary heart disease (CHD). We evaluated whether brachial-ankle pulse wave velocity (baPWV) and augmentation index (AI) are correlated with risk factors of CHD. All of the 528 participants (270 males and 258 females) of this study were healthy workers aged from 36 to 69 (mean age: $47.9 \pm 8.1$ years). The Framingham Risk Score (FRS) showed a good correlation with baPWV ( $r=0.53$, $\mathrm{p}<0.01)$, indicating that FRS is applicable as an index of arterial stiffness also in Japanese. Blood pressures were well controlled in patients with medical treatment for hypertension; however, vessels remained relatively still stiff, while the AI was considerably low. Multivariate linear regression analysis showed that factors of such as FRS, body mass index, alcohol consumption, and AI P75 were significantly correlated with baPWV. (134 words) 
Determinants of baPWV Mitani S

Introduction

Hypertension, hypercholesterolemia and smoking are the three biggest risk factors of coronary heart disease (CHD) according to the Framingham Study. Additionally, the Framingham Risk Score (FRS) was developed to predict the 10-year risk of CHD in a person aged 30-74 years without atherosclerotic CHD $(1,2)$. It is uncertain whether FRS is also applicable to Japanese, because it is based on Euro-American data.

Mortality from CHD in Japan is considerably lower than Euro-American countries.

Studies are needed to assess whether FRS can predict the risk of CHD also in Japanese.

At present, the three most common causes of death in Japan are cancer, heart disease, and stroke, leading to $273.5,143.7$ and 97.2 deaths per 100,000 person years, respectively, according to data compiled by The Health, Labour and Welfare Ministry, on the Population Survey Report 2009 Japan (3). The prevention of these diseases is the most important issue in public health in Japan as well as the rest of the world.

These risk factors of CHD such as age, blood pressure, serum cholesterol, diabetes mellitus and obesity are also risk factors of arterial stiffness. Arterial stiffness is associated with a number of deleterious cardiovascular conditions and has been identified as an independent risk factor for CHD. 
Determinants of baPWV Mitani S

Although pulse wave velocity (PWV) of the aorta is being used as the standard measure for arterial stiffness in Western countries, it can be measured in some other arterial regions, such as heart-carotid (hc), heart-brachial (hb), and femoral-ankle (fa) segments. The most frequently studied index to date among a variety of PWV measures is the carotid-femoral pulse wave velocity (cfPWV).

In Japan and other Asian countries, the brachial-ankle PWV (baPWV) is now widely utilized, because it can be measured easily and noninvasively. The baPWV is applicable as an indicator of arterial stiffness, which is the case in cfPWV (4). The purpose of this study was to evaluate determinants of baPWV in the Japanese population.

Methods

This was a sub-analysis of data of the Japan Multi-institutional Collaborative Cohort (J-MICC) Study in Kyoto conducted by Kyoto Prefectural University of Medicine. All of the 528 participants ( 270 males and 258 females) of this study were general healthy workers of local government offices and companies aged from 36 to 69 and inhabitants of Kyoto Prefecture. Those whose residential addresses were not in Kyoto Prefecture were excluded from this study, because of epidemiological follow-up research. They 
Determinants of baPWV Mitani S

participated in this study after receiving an adequate explanation of the study and signing an informed consent form at a routine annual health check in their workplaces. The present study was conducted from July to December 2009. Measurements of blood pressure, baPWV and augmentation index (AI) were conducted after the subjects had rested in the siting position for at least five minutes in the morning, and subjects were not allowed to smoke before the measurement. All measurements were conducted on the annual health check in the work place of the participants. Each of baPWV (S.M.) and AI (E.O.) measurements was done by the same investigators. After the completion all measurements, each participant got a detailed briefing on the results from a physician. This study consisted of subjects with a health check including serum and urine examinations, questionnaire, and blood pressure, baPWV and AI as original items of the Kyoto survey. This study was approved by the ethics committee of Kyoto Prefectural University of Medicine (E-267).

\section{J-MICC Study}

The J-MICC Study is a cohort study that was started in 2005 to examine gene-environment interactions in lifestyle-related diseases, especially cancers (5). This study was supported by a research grant for Scientific Research on Special Priority 
Determinants of baPWV Mitani S

Areas of Cancer from the Japanese Ministry of Education, Culture, Sports, Science and Technology $(6,7)$. The J-MICC Study group is composed of 10 cohorts surveyed by 10 independent research teams $(8,9)$. Kyoto Prefectural University of Medicine is one of these 10 independent research teams. The participants of the J-MICC Study completed questionnaire on lifestyle factors and diseases, donated blood samples, and, in addition, underwent an examinations of blood pressure, PWV and AI at the time of the baseline survey.

\section{baPWV measurement}

baPWV was measured using a volume-plethysmographic apparatus (Form/ABI:

Omron-Colin, Kyoto, Japan).

\section{Measurements of blood pressure and AI}

The blood pressure was determined by a single measurement on the right upper arm using the oscillometric method. Immediately after the measurement, the waveform of the left radial artery was recorded for determining AI using an arterial applanation tonometry probe incorporating an array of 40 micropiezo-resistive transducers (HEM-9000AI: Omron-Colin, Kyoto, Japan). 
Determinants of baPWV Mitani S

\section{Definition}

Alcohol consumption was classified into two categories depending on the current status, current drinker and no-drinker, because former-drinkers were few.

The smoking status was classified into three categories of current, former, and never smoked. Physical exercise was classified into three categories: one who did not have exercise habit, one who exercised one to three times a month, and one who exercised more than three times a month. The categories of hypertension were classified according to the staging criterion of the Hypertension Guidelines by the Japanese Society of Hypertension Committee (10): optimum BP as SBP $<120 \mathrm{mmHg}$ and diastolic blood pressure $(\mathrm{DBP})<80 \mathrm{mmHg}$, normal $\mathrm{BP}$ as $120 \mathrm{mmHg}=<\mathrm{SBP}=<139 \mathrm{mmHg}$ and $80 \mathrm{mmHg}=<\mathrm{DBP}=<89 \mathrm{mmHg}$, untreated hypertension as systolic $\mathrm{SBP}>=140 \mathrm{mmHg}$ and /or diastolic DBP > $=90 \mathrm{mmHg}$, and treated hypertension

Because the observed AI data are influenced by the heart rate, AI P75 was calculated by setting the heart rate at 75 beats/min for the heart rate compensation.

\section{Framingham risk score}

The experience of the Framingham study population was used to develop an algorithm 
Determinants of baPWV Mitani S

that resulted in a calculated score to predict the 10-year risk of CHD. We calculated the score from the age, sex, smoking status, blood level of total cholesterol (T-Ch), high-density lipoprotein cholesterol (HDL-Ch), and SBP with or without hypertension treatment.

\section{Statistical methods}

All data is expressed as the mean \pm SD. Categorical variables are expressed as a percentage. Pearson's correlation coefficients were used to assess the linear relationship between baPWV and age, SBP, DBP, T-Ch, triglycerides (TG), HDL-Ch, HbA1c, AI P75, body mass index (BMI), and FRS. Differences in each variable between any two groups were evaluated using the t-test. Covariance analysis was also performed to analyze associations of categorized classifications such as alcohol consumption, smoking status, exercise habit, presence of parents' CHD events, and status of hypertension, only in a case in which it was possible to hypothesize parallelism, after assessing the absence of an interaction between each factor and covariates (age and sex). Multiple regression models were used to analyze associations between baPWV and other clinical parameters. P-values less than 0.05 was taken as statistically significant. Analyses were performed using SPSS software package 18 (SPSS, Chicago, IL, USA). 
Determinants of baPWV Mitani S

Results

Table 1 shows the characteristics of this study population. The mean age of the subjects was $47.9 \pm 8.1$ (male: $47.7 \pm 8.5$, female: $48.1 \pm 7.8$ ) years. There were significant differences between males and females, with respect to the height, SBP, DBP, T-ch, TG, HDL-ch, AI P75, baPWV and FRS. Males generally had a poor cardiovascular risk profile compared to females, characterized by a higher BP, higher T-Ch and lower HDL-Ch, higher TG, higher baPWV, higher BMI, and higher FRS. baPWV had a strong correlation with the age $(\mathrm{r}=0.51), \mathrm{SBP}(\mathrm{r}=0.65), \mathrm{DBP}(\mathrm{r}=0.66)$, and FRS $(\mathrm{r}=0.53)$, and a weak correlation with TG $(r=0.30)$, BMI $(r=0.28)$ and AI P75 (r=0.26). (Table 2)

Fig.1 reveals that baPWV was significantly correlated with FRS (total: $r=0.53, p<0.01$, for male: $\mathrm{r}=0.40, \mathrm{p}<0.01$, for female: $\mathrm{r}=0.65, \mathrm{p}<0.01$ ).

Covariance analysis showed the relations among baPWV, AI P75, alcohol consumption, smoking status, exercise habit, presence of parents' CHD events, and status of hypertension (Table 3-a, and 3-b). Regarding baPWV, there were significant differences in exercise habit adjusted by age and $\operatorname{sex}(\mathrm{F}=3.55, \mathrm{p}=0.03)$ and the status of hypertension adjusted by sex $(\mathrm{F}=4.09, \mathrm{p}=0.04)$, after assessing the absence of an interaction. Those who exercised more than three times a month showed a lower 
Determinants of baPWV Mitani S

baPWV than those who did one or three times and those who did not have exercise habit in subgroup analysis. As for hypertension, there was an interaction between hypertension and age; thus, covariance analysis was conducted to compare the hypertension status with baPWV using only sex as a covariant. Regarding BP, the BP values of those who received treatment (SBP: $136.4 \mathrm{mmHg}$, DBP: $86.7 \mathrm{mmHg}$ ) were lower than those who did not (SBP: $141.7 \mathrm{mmHg}$, DBP: $93.4 \mathrm{mmHg}$ ). However, those with anti-hypertensive treatment showed a higher baPWV than the others. (Table 3-a) On the other hand, regarding AI P75, there were significant differences in alcohol consumption $(\mathrm{F}=12.9, \mathrm{p}<0.01)$, smoking status $(\mathrm{F}=7.20, \mathrm{p}<0.01)$ and the status of hypertension $(\mathrm{F}=5.67, \mathrm{p}<0.01)$. Those who were current drinkers and those who had never smoked showed a significantly lower AI P75. Those with hypertension without treatment showed a higher AI P75 than those with an optimum and normal BP; however, there were no significant differences between those with anti-hypertensive treatment and the others. Those whose parents had experienced CHD events were 139 persons, those whose parents had experienced no events were 305 persons, and those who did not know comprised 88 persons. Regarding both baPWV and AI P75, there were no significant differences between the presence and absence of parents' CHD events. (Table 3-b) 


\section{Determinants of baPWV Mitani S}

We conducted multivariate linear regression analysis with FRS, BMI, AI P75, alcohol consumption, exercise habit and presence of parents' CHD events. All of the variables were involved, because no correlation coefficient of a variable was over 0.9 after checking a correlation matrix table. The results of multivariable linear regression stepwise analysis are shown in Table 4 . The result of ANOVA was significant, and $\mathrm{R}^{2}$ was 0.33 . The Durbin-Watson ratio was 2.06 , and there was no separate predictive value over $\pm 3 \mathrm{D}$ against the actual observed value. Thus, factors of FRS, BMI, alcohol consumption and AI P75 were related to baPWV.

Discussion

The Korean data which has the same Asian population with Japanese reported that cfPWV was associated with the CVD risk as represented by FRS $(r=0.417, p<0.01)$ (11). Tanaka et al. also reported that baPWV and cfPWV were closely associated with blood pressure, FRS and pre-existing CHD in Japanese. In their researches, the correlation coefficient of baPWV and FRS ( $\mathrm{r}=0.63$ ) was higher than that of cfPWV and FRS ( $\mathrm{r}=0.48) .(12)$ Many previous studies reported a correlation between baPWV and FRS, and our study also confirmed this. The stiffness of vessels showed by baPWV had a good correlation with lifestyle parameters being risk factors showed by FRS. 
Determinants of baPWV Mitani S

However, only FRS did not determine the stiffness of vessels. There were a lot of relative factors on baPWV showed in Table 2. It was a fact that FRS shows a strong correlation with baPWV, however, age, gender and BP tended to be stronger factors correlated with baPWV. Although diabetes was reported to be an evident factor of CHD and stroke in NIPPON DATA (13), our study showed that there was no statistically significant correlation between $\mathrm{HbA1c}$ and baPWV ( $\mathrm{r}=-0.03, \mathrm{p}=\mathrm{ns})$. Our data was an accurate assessment regarding the correlation between the abnormal glucose tolerance and the stiffness of vessels, while NIPPON DATA addressed the mortality and occasional blood glucose level. On the other hand, another study reported that diabetes mellitus had a significant correlation with baPWV (14). Thus, further research will be needed to clarify the relation between diabetes and baPWV.

Covariance analysis showed conflicting results regarding alcohol consumption and smoking between baPWV and AI P75. Although baPWV reflects the stiffness of vessels, AI does the ratio between reflected and ejected waves (15). The bigger a reflected wave, and the shorter the returning time of a reflected wave, the bigger the value of AI. AI denotes systolic overload of the left ventricle (impedance). In the presence study, smoking status and alcohol consumption affected not baPWV but AI 75. Current drinker and never-smoker revealed significantly milder overload of the 
Determinants of baPWV Mitani S

left ventricle than their counterparts. However, multivariate linear regression analysis

showed a significant effect of alcohol consumption on baPWV, which is interpreted by a

fact that beverages provide protection against an oxidative stress in blood vessels and decrease arterial stiffness (16).

Although our study did not analyze the intensity and kind of exercise, our results showed that exercise with high frequency made blood vessels smoother, and reduced the overload for heart. It has been shown that regular aerobic exercise is associated with reduction in CHD morbidity and mortality (17). Several studies have also shown that aerobic exercise improves endothelium-dependent vasodilation (18). Another study has revealed that moderate aerobic exercise reduces endothelin-1-mediated vasoconstrictor tone (19). These mechanisms allow vessels to be smoother by aerobic exercise. Indeed, some studies reported that exercise caused cfPWV reduction in patients with hypertension (20). Our study supported these findings in a healthy Japanese population.

On the other hand, history of parents' CHD is also reported to be a risk factor of CHD in the USA. Some epidemiological studies including prospective studies reported that the family history was independent of other risk factors $(21,22)$. However, our results were disagreement with above mentioned findings. This may be explained, at least in part, by 


\section{Determinants of baPWV Mitani S}

the fact that questionnaire was badly-phrased one, or general participants could not remember the history of their parents' diseases.

More noteworthy was influence of hypertension. The blood vessels of those who had anti-hypertensive treatment indeed were stiffer as shown in table III-a, however, the overload for heart were lower than those who did not have treatment as shown in table III-b showed.

The difference between arterial stiffness and AI75 may be explained as follows. It is assumed that arterial stiffness is determined by pathological characteristics of large conductance arteries which seem to be stable for a long period regardless of anti-hypertension treatment. In contrast, AI75 is influenced by peripheral arterial tone which is sensitive to anti-hypertensive treatment. These findings suggest that treatment for hypertension is probably useful to prevent the occurrence of CHD. Some previous studies have demonstrated that hypertension is an independent risk factor of CHD even when adjusted by other factors. A dose-response relationship, whereby an increased BP is associated with an increased risk of the development of CHD, could be realized. The Hisayamacho study, which is one of the most famous epidemiological studies in Japan, reported that the extent of arterial stiffness was significantly correlated with SBP of both the start of the study and just prior to death 
Determinants of baPWV Mitani S

However, there may be some complicated mechanisms regarding how increased SBP

leads to CHD occurrence in Japanese. Hirai Y, et al. reported that SBP is used to predict cardiovascular mortality in a farming but not in a fishing community in a 40-year follow up of Japanese cohorts in the Seven Countries Study (24).

Additionally, baPWV had relation with not only FRS but also BMI, alcohol consumption, and AI75 as shown in table IV. The Framingham study reported that obesity was an independent risk factor compared with age, smoking status, total cholesterol levels, SBP, diabetes mellitus and cardiomegaly from their 26-year investigation (25). Although there are numerous studies indicating the correlation between obesity and CHD, FRS does not include the factor of obesity. Our study indicated that BMI was an independent risk factor for arterial stiffness.

\section{Conclusions}

1) The FRS and baPWV show a good correlation. FRS seems to be applicable as an index of arterial stiffness also in Japanese.

2) Blood pressure in patients with anti-hypertensive treatment is well controlled; however, vessels are still stiff, whereas the AI is considerably low. These findings suggest that anti-hypertensive treatment is probably useful to prevent CHD. 
Determinants of baPWV Mitani S

Acknowledgment

This study was supported, in part, by the Ministry of Education, Culture, Sports,

Science and Technology (Nos. 1705018 and 221S0001). Conflicts of interest: None declared.

References

1. Wilson PW, D'Agostino RB, Levy D, et al. Prediction of coronary heart disease using risk factor categories. Circulation. 1998; 97: 1837-47.

2. D'agostino RB Sr, Grundy S, Sullivan LM, Wilson P. Validation of the Framingham coronary heart disease prediction score; Results of a multiple ethnic groups investigation. JAMA. 2001; 286: 180-7.

3. Population Survey Report 2009 Japan [homepage on the Internet] Ministry of Health, Labour and Welfare; 2010 [updated as needed; cited 14 September 2011]. Available from:

http://www.mhlw.go.jp/toukei/saikin/hw/jinkou/suii09/deth1.html

4. Tanaka T, Munakata M, Kawano Y, Ohishi M, Shoji T, Sugawara J, et al.

Comparison between carotid-femoral and brachial-ankle pulse wave velocity as 
Determinants of baPWV Mitani S

measures of arterial stiffness. J Hypertension. 2009; 27: 2022-7.

5. The J-MICC Study Group. The Japan Multi-Institutional Collaborative Cohort Study (J-MICC Study) to detect geneenvironment interactions for cancer. Asian Pac J Cancer Prev. 2007; 8: 317-23.

6. Naito M, Eguchi H, Okada R, Ishida Y, Nishio K, Hishida A, et al. Controls for monitoring the deterioration of stored blood samples in the Japan Multi-Institutional Collaborative Cohort Study (J-MICC Study). Nagoya J Med Sci. 2008; 70: 107-15. 7. Wakai K, Hamagima N, Okada R, Morita E, Hishida A, Kawai S, et,al. Profile of participants and genotype distribution of 108 polymorphisms in a cross-sectional study of associations of genotypes with lifestyle and clinical factors: a project in the Japan Multi-Institutes Collaborative Cohort (J-MICC) study. J Epidemiol. 2011; 21: 223-35. 8. Asai Y, Naito M, Suzuki M, Tomoda A, Kuwabara M, Fukada Y, et al. Baseline data of Shizuoka area in the Japan Multi-Institutional Collaborative Cohort Study (J-MICC Study). Nagoya J Med Sci. 2009;71: 137-44.

9. Hara M, Higaki Y, Imaizumi T, Taguchi N, Nakamura K, Nanri H, et al. Factors influencing participation rate in a baseline survey of a genetic cohort in Japan. $J$ Epidemiol. 2010; 20: 40-5.

10. Ogihara T, Kikuchi K, Matsuoka H, et al. The Japanese Society of Hypertension 
Determinants of baPWV Mitani S

Guidelines for the Management of Hypertension (JSH 2009). Hypertens Res, 2009; 32:

$3-107$.

11. Song BG, Park JB, Cho SJ, Lee SY, Kim JH, Choi SM, Park JH, Park YH, Choi JO,

Lee SC, Park SW. Pulse wave velocity is more closely associated with cardiovascular

risk than augmentation index in the relatively low-risk population. Heart Vessels. 2009;

24: 413-8.

12. Tanaka H, Munakata M, Kawano Y, Ohishi M, Shoji T, Sugawara J, Tomoyama H,

Yamashita A, Yasuda H, Sawayama T, Ozawa T. Comparison between carotid-femoral

and brachial-ankle pulse wave verbosity as measurement of arterial stiffness. $J$

Hypertens. 2009; 27: 2022-7.

13. NIPPON DATA80 Research Group. Risk assessment chart for death from

cardiovascular disease based on a 10-year follow-up study of a Japanese representative population - NIPPON DATA80-. Circ J. 2006; 70; 1249-55.

14. Tsuchikura S, Shoji T, Kimoto E, Shinohara K, Hatsuda S, Koyama H, et al.

Brachial-ankle pulse wave velocity as an index of central arterial stiffness. $J$

Atheroscler Thromb. 2010; 17: 658-65.

15. Kelly R, Hayward C, Avolio A, O’Rourke M. Noninvasive determination of age-ralated changes in the human arterial pulse. Circulation. 1989: 80; 
Determinants of baPWV Mitani S

1952-9.

16. Krnic M, Modun D, Budimir D, Gunjaca G, Jajic I, Vokovic J, et al. Comparison of acute effects of red wine, beer and vodka against hyperoxia-induced oxidative stress and increase in arterial stiffness in healthy humans. Atherosclerosis. 2011; 218; 530-5.

17. Powell KE, Thompson PD, Caspersen CJ, Kendrick JS. Physical activity and the incidence of coronary heart disease. Annr Rev Public Health . 1987; 8: 253-87.

18. Higashi Y, Sasaki S, Kurisu S, Yoshimizu A, Sasaki N, Kajiyama G, et al. Regular aerobic exercise augment endothelium-dependent vascular relaxation in normotensive as well as hypertensive subjects: role of endothelium-derived nitric oxide. Circulation 1999; 100: 1194-202.

19. Stauffer BL, Westby CM, Desouza CA. Endothelin-1, aging and hypertension. Curr Opin Cardiol. 2008; 23: 350-5.

20. Collier SR, Kanaley JA, Carthart R Jr, Frechette V, Tobin MM, Hall AK, et al. Effect of 4 weeks of aerobic or resistance exercise training on arterial stiffness, blood flow and blood pressure in pre- and stage-1 hypertensive. J Hum Hypertens. 2008; 22: 678-86.

21. Hopkins PN, Williams RR, Kuida H, Stults BM, Hunt SC, Barlow GK, Ash KO. Family history as an independent risk factor for incident coronary artery disease in a high-risk cohort in Utah. Am J Cardiol. 1988; 62: 703-7. 
Determinants of baPWV Mitani S

22. Hopkins PN, Williams RR. Human genetics and coronary heart disease: a public health perspective. Annu Rev Nutr. 1989; 9: 303-45.

23. Okumiya N, Tanak K, Ueda K, Omae T. Coronary atherosclerosis and antecedent risk factors: pathologic and epidemiologic study in Hisayama, Japan. Am J Cardiol. 1985; 56: 62-6.

24. Hirai Y, JM Geleijnse, Adachi H, Imaizumi T, Kromhout D. Systolic blood pressure predicts cardiovascular mortality in a farming but not in a fishing community - a 40-year follow up of the Japanese cohorts of the Seven Countries Study-. Circ J. 2011; 75: $1890-6$

25. Hubert HB, Feinleib M, McNamara PM, Castelli WP. Obesity as an independent risk factor for cardiovascular disease: a 26-year follow-up of participants in the Framingham Heart Study. Circulation. 1983; 67: 968-77. 
Fig. I . Correlation plots between brachial-ankle pulse wave velocity and Framingham risk score.

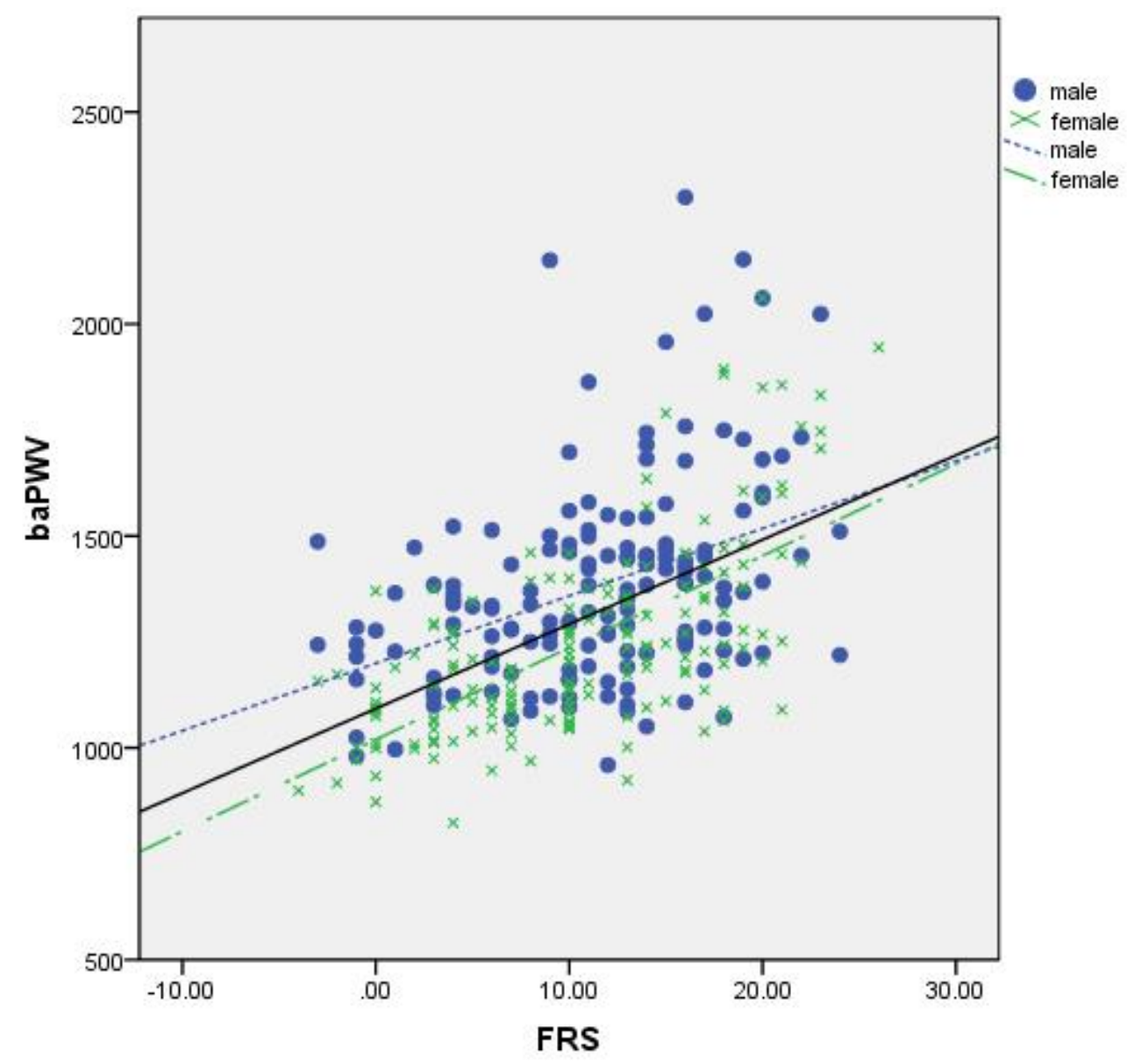

Figure legend

Closed circle means male, cross means female. The dotted line means male $(r=0.40)$, dashed-dotted line means female $(\mathrm{r}=0.65)$, and continuous line means total of male and female ( $\mathrm{r}=0.53)$. baPWV: brachial-ankle pulse wave velocity, FRS: Framingham risk score. 
Table I . Characteristics of the participants

\begin{tabular}{lccccc} 
& Total & Male & Female & t & P value \\
\hline Total number of subjects $(\%)$ & 528 & $270(51.1)$ & $258(48.9)$ & & \\
Age (years) & $47.9 \pm 8.1$ & $47.7 \pm 8.5$ & $48.1 \pm 7.8$ & 0.54 & 0.59 \\
Height $(\mathrm{cm})$ & $163.7 \pm 9.0$ & $170.4 \pm 5.7$ & $156.6 \pm 5.7$ & 27.64 & $<0.01$ \\
SBP $(\mathrm{mmHg})$ & $120 \pm 16$ & $127 \pm 15$ & $115 \pm 16$ & 7.73 & $<0.01$ \\
DBP (mmHg) & $74 \pm 11$ & $78 \pm 11$ & $70 \pm 11$ & 7.59 & $<0.01$ \\
T-ch (mg/dL) & $204 \pm 36$ & $198 \pm 37$ & $210 \pm 35$ & 2.88 & $<0.01$ \\
TG (mg/dL) & $106 \pm 64$ & $129 \pm 67$ & $88 \pm 55$ & 7.09 & $<0.01$ \\
HDL-ch (mg/dL) & $64 \pm 16$ & $57 \pm 14$ & $69 \pm 16$ & 8.95 & $<0.01$ \\
HbA1c $(\%)$ & $5.0 \pm 0.5$ & $5.0 \pm 0.6$ & $5.1 \pm 0.5$ & 1.31 & 0.19 \\
HR (beats/min) & $66 \pm 11$ & $67 \pm 11$ & $66 \pm 11$ & 0.14 & 0.89 \\
AI P75 & $72.4 \pm 14.4$ & $66.7 \pm 13.6$ & $78.3 \pm 12.6$ & 10.10 & $<0.01$ \\
baPWV (cm/sec) & $1,305 \pm 230$ & $1,367 \pm 220$ & $1,243 \pm 14$ & 6.40 & $<0.01$ \\
BMI $\left(\mathrm{kg} / \mathrm{m}^{2}\right)$ & $22.5 \pm 2.9$ & $23.4 \pm 2.8$ & $21.6 \pm 2.8$ & 7.34 & $<0.01$ \\
FRS & $10.9 \pm 6.5$ & $11.4 \pm 6.0$ & $10.5 \pm 6.8$ & 1.21 & $<0.01$
\end{tabular}

SBP: systolic blood pressure

DBP: diastolic blood pressure

T-ch: total cholesterol

TG: triglyceride

HDL-ch: high-density lipoprotein cholesterol

HR: heart rate

AI P75: augmentation index pulse 75

baPWV: brachial-ankle pulse wave velocity

BMI: body mass index

FRS: Framingham risk score 
Table II. Correlations between factors related to baPWV

\begin{tabular}{|c|c|c|c|c|c|c|c|c|c|c|}
\hline & Age & SBP & DBP & TG & T-ch & HDL-ch & HbAlc & AI P75 & BMI & FRS \\
\hline baPWV & $0.51^{* *}$ & $0.65 * *$ & $0.66^{* *}$ & $0.30^{*}$ & 0.08 & $-0.16^{*}$ & -0.03 & $0.26^{*}$ & $0.28 *$ & $0.53^{* * *}$ \\
\hline Age & & $0.27 *$ & $0.26^{*}$ & $0.14 *$ & $0.18^{*}$ & -0.07 & 0.04 & $0.41 *$ & $0.11 *$ & $0.81 *$ \\
\hline SBP & & & $0.80^{*}$ & $0.30^{*}$ & 0.03 & $-0.19^{*}$ & $-0.16^{*}$ & $0.15^{*}$ & $0.40^{*}$ & $0.37 *$ \\
\hline DBP & & & & $0.29 *$ & 0.08 & $-0.17 *$ & -0.08 & $0.19^{*}$ & $0.37 *$ & $0.35^{*}$ \\
\hline TG & & & & & $0.15^{*}$ & $-0.48^{*}$ & $-0.17^{*}$ & -0.05 & $0.40^{*}$ & $0.31 *$ \\
\hline T-ch & & & & & & $0.24 *$ & 0.08 & $0.12^{*}$ & 0.09 & $0.55^{*}$ \\
\hline HDL-ch & & & & & & & 0.08 & $0.14 *$ & $-0.40 *$ & $-0.12 *$ \\
\hline $\mathrm{HbA1c}$ & & & & & & & & $0.18 *$ & -0.05 & 0.05 \\
\hline AI P75 & & & & & & & & & $-0.10^{*}$ & $0.36^{*}$ \\
\hline BMI & & & & & & & & & & $0.28^{*}$ \\
\hline
\end{tabular}

$* *: \mathrm{p}<0.01, *: \mathrm{p}<0.05$ 
Table III-a. Covariance analysis of baPWV adjusted by age and sex

N (\%) Estimated baPWV Adjusted b y F value P-value

Alcohol consumption

$\begin{array}{llllll}\text { Current drinker } & 310(58.4) & 1,308 \pm 11 & \text { Age and sex } & 0.05 & 0.82 \\ \text { No-drinker / past-drinker } & 221(41.6) & 1,304 \pm 13 & & \end{array}$

Smoking

$\begin{array}{lcc}\text { Current smoker } & 108(20.3) & 1,316 \pm 20 \\ \text { Former smoker } & 90(16.9) & 1,314 \pm 21 \\ \text { Never } & 333(62.7) & 1,302 \pm 11\end{array}$

Physical exercise

Nothing
1-3 times/month
More

$111(20.9) \quad 1,349 \pm 18$

$120(22.6) \quad 1,303 \pm 17$

$301(56.6) \quad 1,292 \pm 11$

Presence of parents' CVD event

$\begin{array}{lcc}\text { Yes } & 139(26.1) & 1,320 \pm 16 \\ \text { No } & 305(57.3) & 1,303 \pm 11 \\ \text { Unknown } & 88(16.5) & 1,296 \pm 21\end{array}$

Hypertension

\begin{tabular}{|c|c|c|c|c|}
\hline Optimum BP & $204(38.3)$ & $1,153 \pm 13$ & $\mathrm{Se}$ & 4.09 \\
\hline Normal BP & $156(29.3)$ & $1,318 \pm 14$ & & \\
\hline $\begin{array}{l}\text { Hypertension without } \\
\text { treatment }\end{array}$ & $112(21.1)$ & $1,463 \pm 17$ & & \\
\hline $\begin{array}{l}\text { Hypertension with } \\
\text { treatment }\end{array}$ & $50(9.4)$ & $1,537 \pm 25$ & & \\
\hline
\end{tabular}


Table III-b. Covariance analysis of AI adjusted by age and sex

N (\%) Estimated AI75 Adjusted b y $\quad$ F value P-value

Alcoholl consumption

Current drinker
No-drinker / past-drinker

306

$70.7 \pm 15.1$

Age

$12.9<0.01$

Smoking

$\begin{array}{lcc}\text { Current smoker } & 108(20.3) & 76.6 \pm 1.2 \\ \text { Former smoker } & 90(16.9) & 72.4 \pm 1.3 \\ \text { Never } & 333(62.7) & 71.0 \pm 0.7\end{array}$

Physical exercise

Nothing

$74.3 \pm 1.2$

Age and sex

1.87

0.16

1-3 times/month

118

$72.7 \pm 1.1$

More

298

$71.6 \pm 0.7$

Presence of parents' CVD event

Yes

Unknown

Hypertension

\section{Optimum BP}

Normal BP

Hypertension without

treatment

Hypertension with

treatment
$70.1 \pm 0.9$

Age and sex

5.67

$<0.01$
$76.2 \pm 1.1$

$72.8 \pm 1.7$ 
Table IV. Multivariable liner regression analysis between baPWV and risk factors

\begin{tabular}{cccccc} 
& $\begin{array}{c}\text { Unstandardized } \\
\text { coefficient }\end{array}$ & $\begin{array}{c}\text { Standardized } \\
\text { coefficient }\end{array}$ & p-value & \multicolumn{2}{c}{$95 \%$ CI } \\
\hline Invariable & 694.86 & 119.10 & $<0.01$ & 460.51 & 929.22 \\
FRS & 15.88 & 2.01 & $<0.01$ & 11.93 & 19.83 \\
BMI & 14.48 & 4.11 & $<0.01$ & 6.41 & 22.56 \\
Alcohol & -33.30 & 11.42 & $<0.01$ & -55.78 & -10.83 \\
AI P75 & 2.44 & 0.87 & $<0.01$ & 0.74 & 4.15 \\
\hline
\end{tabular}

CI: confidence interval

Abbreviations are same as in Table I . 